\title{
Review Article \\ Lower Level of Bacteroides in the Gut Microbiota Is Associated with Inflammatory Bowel Disease: A Meta-Analysis
}

\author{
Yingting Zhou and Fachao Zhi \\ Guangdong Province Key Laboratory of Gastroenterology, Department of Gastroenterology, Nanfang Hospital, Guangzhou, China \\ Correspondence should be addressed to Fachao Zhi; zhifc41532@163.com
}

Received 17 May 2016; Accepted 9 August 2016

Academic Editor: Yutao Yan

Copyright ( 2016 Y. Zhou and F. Zhi. This is an open access article distributed under the Creative Commons Attribution License, which permits unrestricted use, distribution, and reproduction in any medium, provided the original work is properly cited.

\begin{abstract}
Background and Aims. Multiple studies have reported associations between inflammatory bowel disease (IBD) and the flora disequilibrium of Bacteroides. We performed a meta-analysis of the available data to provide a more precise estimate of the association between Bacteroides level in the gut and IBD. Methods. We searched PubMed/MEDLINE, EMBASE, Cochrane Library, Wiley Library, BIOSIS previews, Web of Science, CNKI, and ScienceDirect databases for published literature on IBD and gut microbiota from 1990 to 2016. Quality of all eligible studies was assessed using the Newcastle-Ottawa Quality Assessment Scale (NOS). We compared the level of Bacteroides in IBD patients with that in a control group without IBD, different types of IBD patients, and IBD patients with active phase and in remission. Results. We identified 63 articles, 9 of which contained sufficient data for evaluation. The mean level of Bacteroides was significantly lower in Crohn's disease (CD) and ulcerative colitis (UC) patients in active phase than in normal controls. The level of Bacteroides in remission CD and UC patients was much lower than patients in the control group. Bacteroides level was even lower in patients with CD and UC in active phase than in remission. Conclusions. This analysis suggests that lower levels of Bacteroides are associated with IBD, especially in active phase.
\end{abstract}

\section{Introduction}

Inflammatory bowel disease (IBD) which includes Crohn's disease (CD), ulcerative colitis (UC), and indeterminate colitis (IC) [1] is a chronic relapsing inflammatory disorder of the gastrointestinal tract [2] of unclear etiology. One hypothesis is that the inflammation results from altered microbiota in a genetically susceptible host [3].

There are 160 major bacteria among the 1,000 to 1,150 species of bacteria which colonize the human intestinal tract. Two dominant microflora in the human distal gut, Bacteroides and Firmicutes phyla, account for $90 \%$ of the bacterial flora [4]. Microflora which play an important physiological role in the adult colon are Bacteroides fragilis, Bifidobacterium, Bacteroides, and a variety of anaerobic Gram-positive cocci. Intestinal flora in healthy individuals can demonstrate significant variety. As individuals age, their intestinal microbial flora tend to become more similar [5]. There is a healthy balance of microflora in the gastrointestinal tract in normal individuals. This balance is disrupted in disease.
Many studies have related Bacteroides to the development of IBD. Bacteroides is a Gram-negative, nonspore forming, obligate anaerobic bacteria normally found in the human intestines, mouth, upper respiratory tract, and genital tract [6]. Bacteroides expresses polysaccharide $\mathrm{A}$, which can induce regulatory $\mathrm{T}$ cell growth and cytokine expression that are protective against colitis [7].

We performed a meta-analysis of case-control studies to assess role of Bacteroides in IBD.

\section{Methods}

2.1. Data Sources and Searches. A literature search was performed using the Preferred Reporting Items for Systematic Reviews and Meta-Analysis (PRISMA) criteria [8] and MetaAnalysis of Observational Studies in Epidemiology (MOOSE) [9] in the following databases: PubMed/MEDLINE, Cochrane Library, ScienceDirect, EMBASE, BIOSIS previews, Web of Science, CNKI, and Wiley Library. The search period was January 1, 1990, through June 30, 2016. Multiple 
search strategies were used and keywords were used in preference to MeSH terms to increase the sensitivity of our search. The following key words were used: inflammatory bowel disease, IBD, Crohn's disease, CD, ulcerative colitis, UC, indeterminate colitis, IC, flora disequilibrium, and Bacteroides.

An expanded search was done using Google Scholar and by contacting authors of selected articles. Conference abstracts and the bibliography of selected articles were also selected to assure that no relevant studies were missed. If two or more studies shared study populations or more than one article reported the same clinical trial, the publication with more information was selected. Professors Bo Jiang and Yang Bai from the Institute of Digestive Diseases, Nanfang Hospital were contacted as the local experts in the field of gut microbiota.

2.2. Study Selection. Studies included for analysis (a) were case-control studies; (b) had the Bacteroides level in the intestines determined; (c) were published articles or meeting abstracts; (d) compared the level of Bacteroides in patients with IBD and without IBD; (e) were published reports with enough data to analyze differences between the IBD and control groups; (f) were published in English or Chinese.

Two reviewers (YT.Z and FC.Z) independently assessed the quality of all eligible studies using the Newcastle-Ottawa Quality Assessment Scale (NOS) (http://www.ohri.ca/programs/clinical_epidemiology/oxford.asp) for case-control studies. The NOS uses a "stars" rating system to judge quality including selection of the study population, comparability, and exposure assessment. Scores were ranged from 0 (the lowest) to 9 (the highest). Studies with a score $\geq 7$ were considered to be of high quality. The quality of each study was awarded stars independently by the same two reviewers (Table 3).

Data was extracted from each qualified study, including the study design, first author's last name, and publication year. Discrepancies between the two authors were dealt with by a consensus meeting with all authors and discussion with our local experts.

2.3. Statistic Analysis. Three primary analyses were performed. Different types of IBD patients' Bacteroides levels were compared with a control group. The level of Bacteroides in patients with CD, UC, and IC were also compared. Patients with active $\mathrm{CD}$ or $\mathrm{UC}$ and patients with $\mathrm{CD}$ or UC in remission were compared. Heterogeneity between studies was assessed using both the $\chi^{2}$ test with a $P$ value $<0.05$ and the inconsistency index $\left(I^{2}\right)$ with a cut-off of $\geq 50 \%$.

Statistical analyses were performed using SPSS version 13.0 (SPSS Corporation, Chicago, USA) and Revman version 5.0 (The Cochrane Collaboration, Oxford, UK).

\section{Results}

63 studies were initially identified. Twenty-one studies were excluded with animal experiments, twenty studies were excluded with irrelevant experiments, and eight reviews were excluded. After that, fourteen studies potentially relevant were further screened. One study was excluded because
Bacteroides was acquired from the gums [10]. Four studies were excluded with data that was inappropriate for analysis [11-14].

Finally, 9 studies evaluating 706 patients met the inclusion criteria (Figure 1) [15-23]. Six articles reported continuous data and three reported dichotomous data (Table 1).

The baseline characteristics of all qualified studies are presented in Table 1. Six studies [15, 19-23] included the mean \pm standard deviation Bacteroides level in different types of IBD patients. This data was used to calculate differences with $95 \%$ CIs. Reports with different methods used to measure Bacteroides (T-RFLD [15, 23], Real-Time Quantitative PCR [22], conventional culture [20], and FISH $[19,21])$ were analyzed separately (Table 2(a)). Three publications $[17,18,22]$ with dichotomous data were analyzed using 95\% CIs (Table 2(b)). Estimate of the study quality scores from NOS system was showed in Table 3.

The Bacteroides level was evaluated for 12 groups, CD versus control group, UC versus control group, IC versus control group, $C D$ versus $U C, C D$ versus $I C, U C$ versus $I C$, active $C D$ versus remission $\mathrm{CD}$, active $\mathrm{UC}$ versus remission $\mathrm{UC}$, active $\mathrm{CD}$ versus control group, and active UC versus control group. The active phase of CD was defined using a Crohn's disease activity index (CDAI) $>150$ and active UC using a Clinical Activity Index $(\mathrm{CAI})>5$. All patients were diagnosed by endoscopy. Continuous data and dichotomous data were analyzed separately.

\subsection{Continuous Data}

3.1.1. IBD Patients versus Control Group. The amount of Bacteroides present in the gut of patients with Crohn's disease was compared to that of a normal control group. Four studies were qualified, with the method of T-RFLP, Real-Time Quantitative PCR, conventional culture, and FISH. Real-Time Quantitative PCR (95\% CI: $-1.94,-0.19, P<0.01$, and Std Mean Difference: -1.42 ) and conventional culture (95\% CI: 0.06, 0.89, $P=0.03$, and Std Mean Difference: 0.47 ) both demonstrated statistically significant difference of Bacteroides between IBD patients and normal healthy patients. Patients with ulcerative colitis were compared to a normal control group for amount of Bacteroides present in the gut. Real-Time Quantitative PCR (95\% CI: $-1.11,0.42, P<0.01$, and Std Mean Difference: -0.77 ) and FISH (95\% CI: 0.46, 1.57, $P<0.01$, and Std Mean Difference: 1.01) both demonstrated statistically significant difference of Bacteroides between IBD patients and in normal controls. No differences were found in patients with indeterminate colitis.

Patients with active Crohn's disease were compared to a normal control group for amount of Bacteroides. One study included with the method of Real-Time Quantitative PCR (95\% CI: $-2.24,-1.01, P<0.01$, and Std Mean Difference: -1.62) demonstrated less Bacteroides present in the gut of active CD patients than in normal controls (Figure 2). Patients with active ulcerative colitis were compared to a normal control group for amount of Bacteroides. Two studies including T-RFLP and FISH test for the overall effect (95\% CI: $-1.12,-0.35, P<0.01$, and Std Mean Difference: -0.68 ) 


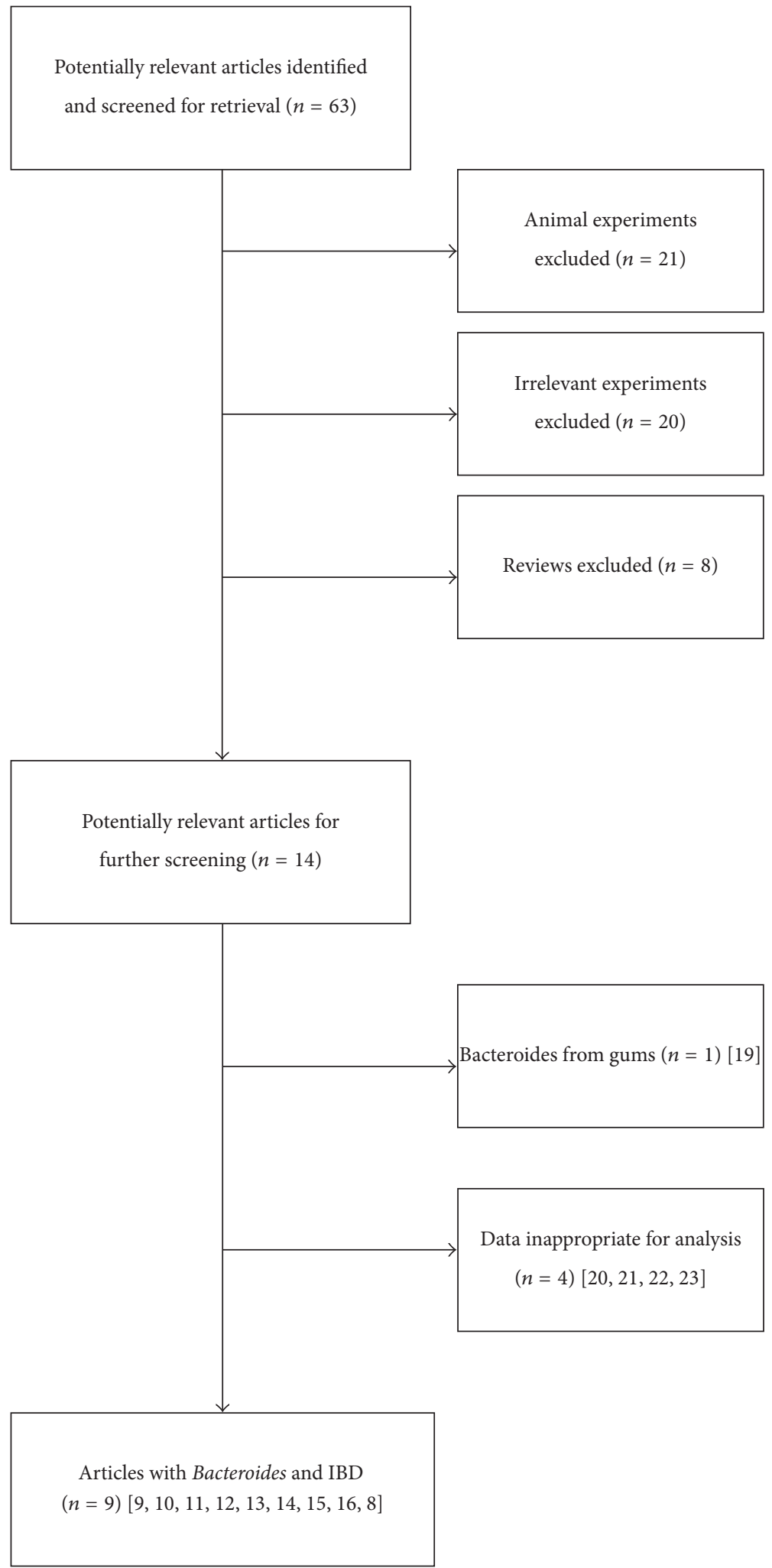

FIGURE 1: Flow diagram of the study selection process for this meta-analysis. 
TABLE 1: Baseline characteristics of the included studies.

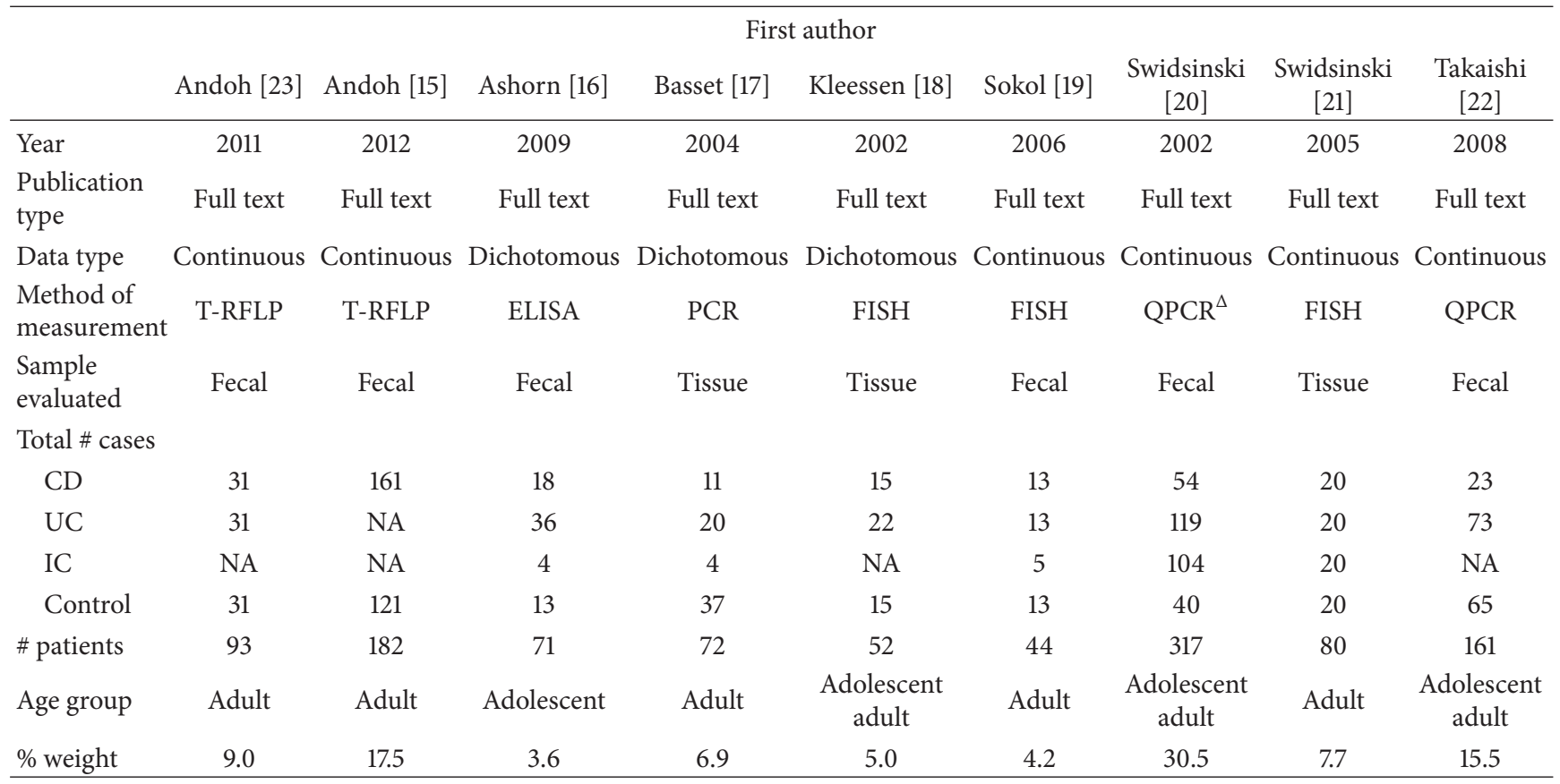

$\mathrm{CD}=$ Crohn's disease, $\mathrm{UC}=$ ulcerative colitis, IC = indeterminate colitis, and NA = no data available; $\Delta:$ QPCR: Real-Time Quantitative PCR.

TABLE 2: (a) Mean Bacteroides levels in different types of IBD patients (continuous data). (b) Bacteroides detection rate in different types of IBD patients (dichotomous data).

(a)

\begin{tabular}{|c|c|c|c|c|c|c|}
\hline & \multicolumn{6}{|c|}{ First author } \\
\hline & Andoh [23] & Andoh [15] & Sokol [19] & $\begin{array}{c}\text { Swidsinski } \\
{[20]}\end{array}$ & Swidsinski [21] & Takaishi [22] \\
\hline Year & 2011 & 2012 & 2006 & 2002 & 2005 & 2008 \\
\hline Method & T-RFLP & T-RFLP & FISH & $\begin{array}{l}\text { Conventional } \\
\text { culture }\end{array}$ & FISH & $\mathrm{QPCR}^{\Delta}$ \\
\hline $\begin{array}{l}\text { Bacteroides levels } \\
\text { in CD patients }\end{array}$ & $0.01 \pm 0.05 * 10^{3}$ & $21.6 \pm 15.4 * 10^{3}$ & $13.8 \pm 11.8$ & $2.0 \pm 5.5$ & $70 \pm 20$ & $8.9 \pm 1.2$ \\
\hline $\begin{array}{l}\text { Bacteroides levels } \\
\text { in UC patients }\end{array}$ & $0.02 \pm 0.12 * 10^{3}$ & NA & $11.7 \pm 11.7$ & $1.6 \pm 10$ & $62 \pm 25$ & $9.3 \pm 1.0$ \\
\hline $\begin{array}{l}\text { Bacteroides levels } \\
\text { in IC patients }\end{array}$ & NA & NA & $36.4 \pm 22.9$ & $0.64 \pm 2.1$ & $40 \pm 19$ & NA \\
\hline $\begin{array}{l}\text { Bacteroides levels } \\
\text { in control group }\end{array}$ & 0 & $16.6 \pm 5.2 * 10^{3}$ & $12.1 \pm 7.0$ & $0.02 \pm 0.05$ & $20 \pm 11$ & $9.9 \pm 0.4$ \\
\hline \multicolumn{7}{|c|}{$\mathrm{CD}=$ Crohn's disease, $\mathrm{UC}=$ ulcerative colitis, $\mathrm{IC}=$ indeterminate colitis, $\underset{(\mathrm{b})}{\operatorname{and}} \mathrm{NA}=$ no data available; $\Delta:$ QPCR: Real-Time Quantitative PCR. } \\
\hline & & \multicolumn{5}{|c|}{ First author } \\
\hline & & \multicolumn{2}{|c|}{ Ashorn [16] } & Basset [17] & & Kleessen [18] \\
\hline Year & & \multicolumn{2}{|c|}{2009} & \multicolumn{2}{|c|}{2004} & 2002 \\
\hline Patients with CD & & \multicolumn{2}{|l|}{$11(18)$} & \multicolumn{2}{|l|}{$3(10)$} & $7(15)$ \\
\hline Patients with UC & & \multicolumn{2}{|l|}{$15(36)$} & \multicolumn{2}{|l|}{$4(18)$} & $8(22)$ \\
\hline Patients with IC ( & & \multicolumn{2}{|l|}{$4(4)$} & \multicolumn{2}{|l|}{$1(4)$} & (NA) \\
\hline Patients in control & up (total) & \multicolumn{2}{|l|}{ (NA) } & \multicolumn{2}{|l|}{$8(32)$} & $6(15)$ \\
\hline
\end{tabular}

$\mathrm{CD}=$ Crohn's disease, $\mathrm{UC}=$ ulcerative colitis $\mathrm{IC}=$ indeterminate colitis, and NA = no data available. 
TABLE 3: Scores from NOS system.

\begin{tabular}{|c|c|c|c|c|c|c|c|c|c|c|}
\hline \multirow{2}{*}{ Studies } & \multicolumn{4}{|c|}{ Comparability } & \multicolumn{2}{|c|}{ Selection } & \multicolumn{3}{|c|}{ Exposure } & \multirow{2}{*}{ Score } \\
\hline & (1) & (2) & (3) & (4) & (1a) & $(1 \mathrm{~b})$ & (1) & (2) & (3) & \\
\hline Andoh et al. 2011 [23] & $*$ & * & * & * & * & * & * & * & * & 9 \\
\hline Andoh et al. 2012 [15] & * & * & * & * & * & * & * & * & - & 8 \\
\hline Ashorn et al. 2009 [16] & * & - & * & * & * & - & * & * & * & 7 \\
\hline Basset et al. 2004 [17] & * & * & * & * & * & - & * & * & * & 8 \\
\hline Kleessen et al. 2002 [18] & * & * & * & * & * & * & * & * & * & 9 \\
\hline Sokol et al. 2006 [19] & * & - & - & * & * & * & - & * & - & 6 \\
\hline Swidsinski et al. 2002 [20] & * & * & * & * & * & - & * & * & * & 8 \\
\hline Swidsinski et al. 2005 [21] & * & * & - & * & * & - & * & * & * & 8 \\
\hline Takaishi et al. 2008 [22] & * & - & - & * & * & $\star$ & - & * & * & 7 \\
\hline
\end{tabular}

Selection: (1) the case definition being adequate; (2) representativeness of the cases; (3) selection of controls, and (4) definition of controls. Comparability: (1a) comparability of cases and controls on the basis of CD, UC, and IC diagnosed of endoscope and pathological section; (1b) study controls for active phase with remission phase with IBD patients with CAI and CDAI. Exposure: (1) ascertainment of exposure; (2) same method of ascertainment for cases and controls; and (3) nonresponse rate.

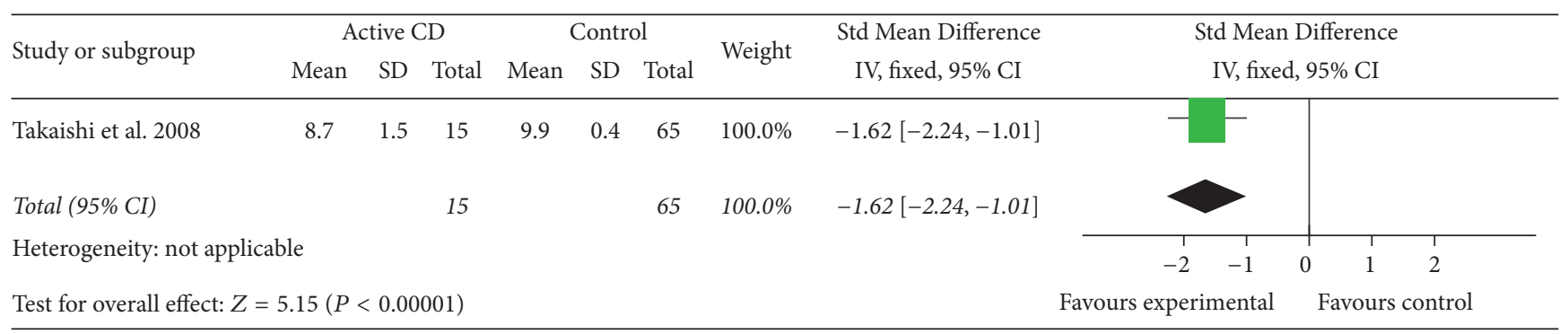

FIGURE 2: Forest plot of active CD versus control group.

demonstrated less Bacteroides present in their gut than in normal controls (Figure 3).

Patients with Crohn's disease in remission were compared to a normal control group for amount of Bacteroides with two studies in Real-Time Quantitative PCR and T-RFLP (Tables 5(a) and 5(b)). Statistically significant difference was found in the amount of Bacteroides of CD in remission and normal control patients (95\% CI: $-1.65,-0.15, P=0.02$, and Std Mean Difference: -0.54$)$. Patients with ulcerative colitis in remission were compared to a normal control group with the same two studies of Real-Time Quantitative PCR and T-RFLP. Patients with UC (95\% CI: $-0.98,-0.09, P=0.02$, and Std Mean Difference: -0.90$)$ had less Bacteroides present in the gut than normal controls.

3.1.2. Different Types of IBD Patients. Both conventional culture (95\% CI: $0.04,0.70, P=0.03$, and Std Mean Difference: 0.37 ) and FISH (95\% CI: $0.1,1.31, P=0.02$, and Std Mean Difference: 0.71 ) identified significantly lower Bacteroides levels of CD patients than in IC patients (Tables 5(c) and 5(d)). No difference was found in the Bacteroides level in the gut of CD versus UC and UC versus IC patients.

3.1.3. IBD Patients with Active Phase and in Remission. Patients with UC in active phase had less Bacteroides in the gut than in remission phase (95\% CI: $-0.98,0.09, P=$ 0.02, and Std Mean Difference: -0.29) (Figure 4). Significant difference was also found in the amount of Bacteroides of active CD and remission CD patients (95\% CI: $-1.48,0.28$, $P<0.01$, and Std Mean Difference: -0.60$)$.

3.2. Dichotomous Data. No difference was found in the amount of Bacteroides of any groups (CD versus control, UC versus control, CD versus UC, CD versus IC, UC versus IC, and IC versus control). There was no evidence of heterogeneity amongst the groups. Comparisons of IC versus control, active $C D$ versus remission $C D$, active $U C$ versus remission $\mathrm{UC}$, active $\mathrm{CD}$ versus control group, active UC versus control group, remission CD versus control group, and remission UC versus control group were not performed as there were not enough patients to perform this analysis (Table 4).

\section{Discussion}

The relationship between intestinal flora and IBD is well described [24, 25]. E. coli [13], Lactobacillus [26, 27], and Bacteroides have been reported to be linked to IBD [28]. Our meta-analysis compared the level of Bacteroides in IBD patients with that in normal controls. Patients with active and inactive disease were also evaluated.

A lower level of Bacteroides was demonstrated with RealTime Quantitative PCR in CD and UC patients than in healthy controls, especially CD and UC patients with active disease. UC patients in remission also had a lower level of 
TABLE 4: Summary of outcomes of included studies with dichotomous data.

\begin{tabular}{lcccc}
\hline Groups compared & OR & $95 \%$ CI & $P$ value & $I^{2}(P)$ \\
\hline CD versus control group & 1.3 & $(0.45,3.77)$ & 0.63 & $0 \%(P=0.98)$ \\
UC versus control group & 0.86 & $(0.33,2.24)$ & 0.75 & $0 \%(P=1.0)$ \\
CD versus UC & 1.8 & $(0.83,3.93)$ & 0.14 & $0 \%(P=0.9)$ \\
CD versus IC & 0.46 & $(0.08,2.69)$ & 0.39 & $0 \%(P=0.32)$ \\
UC versus IC & 0.25 & $(0.05,1.28)$ & 0.1 & $32 \%(P=0.22)$ \\
\hline
\end{tabular}

$\mathrm{CD}=$ Crohn's disease, $\mathrm{UC}=$ ulcerative colitis, and $\mathrm{IC}=$ indeterminate colitis.

\begin{tabular}{|c|c|c|c|c|c|c|c|c|c|c|c|}
\hline \multirow{2}{*}{ Study or subgroup } & \multicolumn{3}{|c|}{ Active UC } & \multicolumn{3}{|c|}{ Control } & \multirow{2}{*}{ Weight } & \multirow{2}{*}{$\begin{array}{l}\text { Std Mean Difference } \\
\text { IV, fixed, 95\% CI }\end{array}$} & \multirow{2}{*}{\multicolumn{3}{|c|}{$\begin{array}{l}\text { Std Mean Difference } \\
\text { IV, fixed, } 95 \% \text { CI }\end{array}$}} \\
\hline & Mean & SD & Total & Mean & $\mathrm{SD}$ & Total & & & & & \\
\hline \multicolumn{12}{|l|}{ 7.4.1 T-RFLP } \\
\hline Andoh et al. 2011 & 0.05 & 0.2 & 15 & 0 & 0 & 31 & $29.2 \%$ & $0.44[-0.19,1.06]$ & & & \\
\hline Subtotal (95\% CI) & & & 15 & & & 31 & $29.2 \%$ & $0.44[-0.19,1.06]$ & & & \\
\hline \multicolumn{12}{|c|}{ Heterogeneity: not applicable } \\
\hline \multicolumn{12}{|c|}{ Test for overall effect: $Z=1.37(P=0.17)$} \\
\hline \multicolumn{12}{|l|}{ 7.4.2 FISH } \\
\hline Takaishi et al. 2008 & 9 & 1.1 & 49 & 9.9 & 0.4 & 65 & $70.8 \%$ & $-1.14[-1.55,-0.74]$ & & & \\
\hline Subtotal (95\% CI) & & & 49 & & & 65 & $70.8 \%$ & $-1.14[-1.55,-0.74]$ & & & \\
\hline \multicolumn{12}{|c|}{ Heterogeneity: not applicable } \\
\hline \multicolumn{12}{|c|}{ Test for overall effect: $Z=5.60(P<0.00001)$} \\
\hline Total (95\% CI) & & & 64 & & & 96 & $100.0 \%$ & $-0.68[-1.02,-0.35]$ & & & \\
\hline \multicolumn{9}{|c|}{ Heterogeneity: $\chi^{2}=17.47, \mathrm{df}=1(P<0.0001) ; I^{2}=94 \%$} & $\begin{array}{ll}1 & \\
-2 & -1\end{array}$ & 0 & 1 \\
\hline \multicolumn{9}{|c|}{ Test for overall effect: $Z=3.97(P<0.0001)$} & Favours experimental & Favour & rs control \\
\hline \multicolumn{12}{|c|}{ Test for subgroup differences: $\chi^{2}=17.47, \mathrm{df}=1(P<0.0001), I^{2}=94.3 \%$} \\
\hline
\end{tabular}

FIGURE 3: Forest plot of active UC versus control group.

\begin{tabular}{|c|c|c|c|c|c|c|c|c|c|}
\hline \multirow{2}{*}{ Study or subgroup } & \multicolumn{3}{|c|}{ Active UC } & \multicolumn{3}{|c|}{ Remission UC } & \multirow{2}{*}{ Weight } & Std Mean Difference & \multirow{2}{*}{$\begin{array}{c}\text { Std Mean Difference } \\
\text { IV, fixed, 95\% CI }\end{array}$} \\
\hline & Mean & SD & Total & Mean & SD & Total & & IV, fixed, 95\% CI & \\
\hline
\end{tabular}

7.2.1 T-RFLP

Andoh et al. 2011

$\begin{array}{lllllll}0.05 & 0.2 & 15 & 0 & 0 & 18 & 31.5 \%\end{array}$

$0.36[-0.33,1.05]$

Subtotal $(95 \%$ CI)

$15 \quad 18 \quad 31.5 \%$

$0.36[-0.33,1.05]$

Heterogeneity: not applicable

Test for overall effect: $Z=1.03(P=0.30)$

\subsubsection{QPCR}

$\begin{array}{lllllllll}\text { Takaishi et al. } 2008 & 9 & 1.1 & 49 & 9.6 & 0.8 & 29 & 68.5 \% & -0.59[-1.06,-0.13] \\ \text { Subtotal }(95 \% \text { CI) } & & & 49 & & & 29 & 68.5 \% & -0.59[-1.06,-0.13]\end{array}$

Heterogeneity: not applicable

Test for overall effect: $Z=2.48(P=0.01)$

Total (95\% CI)

Heterogeneity: $\chi^{2}=5.04, \mathrm{df}=1(P=0.02) ; I^{2}=80 \%$

Test for overall effect: $Z=1.48(P=0.14)$

Test for subgroup differences: $\chi^{2}=5.04, \mathrm{df}=1(P=0.02), I^{2}=80.2 \%$

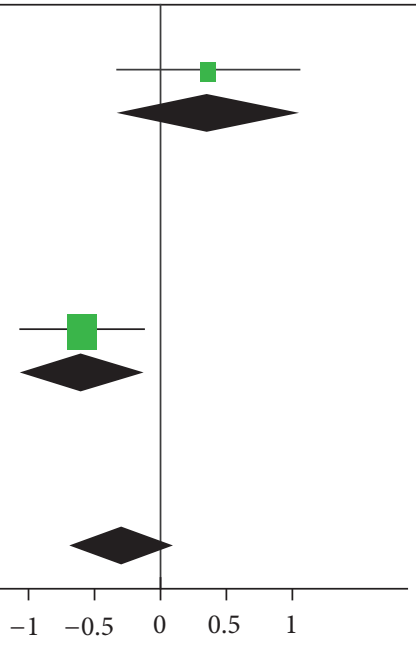

Favours experimental Favours control

FIGURE 4: Forest plot of UC in active phase versus in remission phase. 
TABLE 5: (a) Summary of outcomes of included studies with continuous data (method: T-RFLP). (b) Summary of outcomes of included studies with continuous data (method: $\mathrm{QPCR}^{\Delta}$ ). (c) Summary of outcomes of included studies with continuous data (method: conventional culture). (d) Summary of outcomes of included studies with continuous data (method: FISH).

(a)

\begin{tabular}{|c|c|c|c|c|}
\hline & Standard Mean Difference & $95 \% \mathrm{CI}$ & $P$ value & $I^{2}(P)$ \\
\hline CD versus control group & 0.23 & $(-0.42,0.88)$ & 0.48 & NA \\
\hline UC versus control group & 0.23 & $(-0.27,0.73)$ & 0.36 & NA \\
\hline $\mathrm{CD}$ versus $\mathrm{UC}$ & -0.11 & $(-0.61,0.39)$ & 0.67 & NA \\
\hline ***Active UC versus remission UC & 0.36 & $(-0.33,1.05)$ & 0.30 & NA \\
\hline Active UC versus control group & 0.44 & $(-0.19,1.06)$ & 0.17 & NA \\
\hline
\end{tabular}

$\mathrm{CD}=$ Crohn's disease, $\mathrm{UC}=$ ulcerative colitis, $\mathrm{IC}=$ indeterminate colitis, and $\mathrm{NA}=$ not available; ${ }^{* * *}$ active $\mathrm{UC}: \mathrm{CAI}>5$.

(b)

\begin{tabular}{|c|c|c|c|c|}
\hline & Standard Mean Difference & $95 \% \mathrm{CI}$ & $P$ value & $I^{2}(P)$ \\
\hline CD versus control group & -1.42 & $(-1.94,-0.19)$ & $P<0.001$ & NA \\
\hline UC versus control group & -0.77 & $(-1.11,-0.42)$ & $P<0.001$ & $\mathrm{NA}$ \\
\hline $\mathrm{CD}$ versus $\mathrm{UC}$ & -0.38 & $(-0.85,0.09)$ & 0.12 & NA \\
\hline${ }^{* *}$ Active $\mathrm{CD}$ versus remission $\mathrm{CD}$ & -0.60 & $(-1.48,0.28)$ & $P<0.01$ & NA \\
\hline${ }^{* * *}$ Active UC versus remission UC & -0.29 & $(-0.98,-0.09)$ & 0.02 & NA \\
\hline Active CD versus control group & -1.62 & $(-2.24,-1.01)$ & $P<0.001$ & NA \\
\hline Active UC versus control group & -0.68 & $(-1.02,-0.35)$ & $P<0.01$ & NA \\
\hline Remission CD versus control group & -0.54 & $(-1.65,0.15)$ & 0.02 & NA \\
\hline Remission UC versus control group & -0.90 & $(-0.98,-0.09)$ & 0.02 & NA \\
\hline
\end{tabular}

$\mathrm{CD}=$ Crohn's disease, $\mathrm{UC}=$ ulcerative colitis, $\mathrm{IC}=$ indeterminate colitis, and NA = not available; ${ }^{* *}$ active $\mathrm{CD}: \mathrm{CDAI}>150 ;{ }^{* * *}$ active $\mathrm{UC}: \mathrm{CAI}>5 ; \Delta: \mathrm{QPCR}$ : Real-Time Quantitative PCR.

(c)

\begin{tabular}{|c|c|c|c|c|}
\hline & Standard Mean Difference & $95 \% \mathrm{CI}$ & $P$ value & $I^{2}(P)$ \\
\hline $\mathrm{CD}$ versus control group & 0.47 & $(0.06,0.89)$ & 0.03 & NA \\
\hline UC versus control group & 0.18 & $(-0.18,0.54)$ & 0.32 & NA \\
\hline CD versus UC & 0.04 & $(-0.28,0.37)$ & 0.78 & NA \\
\hline CD versus IC & 0.37 & $(0.04,0.70)$ & 0.03 & NA \\
\hline UC versus IC & 0.13 & $(-0.13,0.39)$ & 0.34 & NA \\
\hline IC versus control group & 0.34 & $(-0.02,0.71)$ & 0.07 & NA \\
\hline
\end{tabular}

$\mathrm{CD}=$ Crohn's disease, $\mathrm{UC}=$ ulcerative colitis, $\mathrm{IC}=$ indeterminate colitis, and $\mathrm{NA}=$ not available.

(d)

\begin{tabular}{|c|c|c|c|c|}
\hline & Standard Mean Difference & $95 \% \mathrm{CI}$ & $P$ value & $I^{2}(P)$ \\
\hline CD versus control group & 0.17 & $(-0.6,0.94)$ & 0.67 & NA \\
\hline UC versus control group & 1.01 & $(0.46,1.57)$ & $P<0.001$ & $93 \%(P<0.001)$ \\
\hline CD versus UC & 0.17 & $(-0.60,0.94)$ & 0.66 & NA \\
\hline CD versus IC & 0.71 & $(0.1,1.31)$ & 0.02 & $94 \%(P<0.001)$ \\
\hline UC versus IC & 0.37 & $(-0.2,0.95)$ & 0.20 & $92 \%(P<0.001)$ \\
\hline IC versus control group & -0.54 & $(-1.13,0.06)$ & 0.08 & $94 \%(P<0.001)$ \\
\hline
\end{tabular}

$\mathrm{CD}=$ Crohn's disease, $\mathrm{UC}=$ ulcerative colitis, $\mathrm{IC}=$ indeterminate colitis, and $\mathrm{NA}=$ not available.

Bacteroides than controls. Patients with active UC had lower Bacteroides levels than patients in remission.

$\mathrm{CD}$ and UC patients had a higher level of Bacteroides in the gut than control patients in FISH and conventional culture studies. These methods also demonstrated a higher level of Bacteroides in the gut of CD patients than in IC patients. The level of Bacteroides in active IC patients was not reported.

CD and UC patients had lower levels of Bacteroides than normal controls in Real-Time Quantitative PCR studies, but higher levels of Bacteroides than normal controls in FISH and conventional culture studies. Real-Time Quantitative PCR [22] fecal samples were mostly obtained from Asian patients, while FISH and conventional culture fecal samples were mostly from European patients. This finding suggests Bacteroides levels may be different in different ethnic groups.

Heterogeneity was found using a fixed effects model in the pooled UC versus control group, IC versus control group, and $\mathrm{CD}$ versus IC group. All these reports used FISH to 
determine Bacteroides levels. Similar findings were observed using a random effects model. Heterogeneity was attributed to two European reports, that of Sokol et al. [19] (using fecal samples) and that of Swidsinski et al. [21] (using tissue samples).

A meta-analysis is inherently limited by the studies included. In this study, many of the reports were descriptive in nature and had small sample sizes. Only two of the 6 studies reported continuous data that could be used to calculate Bacteroides levels in patients with active disease and disease in remission. Therefore, significant heterogeneity was found between the studies when data was pooled. We thought the source of the heterogeneity maybe resulted from the difference of methods to determine Bacteroides and the age. While the included publications used different methods and had small sample sizes and different patient populations, they all reached the conclusion that Bacteroides level was associated with IBD, especially in patients with active disease.

This meta-analysis of observation studies supports the finding of low Bacteroides levels in patients with IBD. Despite the differences in methods, our meta-analysis demonstrated that low levels of Bacteroides were present in IBD patients with active disease. This finding may be useful in the treatment and the etiology research of IBD patients. Prospective trials are needed to confirm the results of this meta-analysis.

\section{Competing Interests}

There is no conflict of interests related to this paper.

\section{Acknowledgments}

The authors thank Drs. Bo Jiang and Yang Bai from the Nanfang Hospital for providing expert opinion in the selection of the relevant articles and reviewing the results of this metaanalysis.

\section{References}

[1] M. T. Osterman, R. Kundu, G. R. Lichtenstein, and J. D. Lewis, "Association of 6-thioguanine nucleotide levels and inflammatory bowel disease activity: a meta-analysis," Gastroenterology, vol. 130, no. 4, pp. 1047-1053, 2006.

[2] R. Srinivasan and A. K. Akobeng, "Thalidomide and thalidomide analogues for induction of remission in Crohn's disease," Cochrane Database of Systematic Reviews, no. 2, Article ID CD007350, 2009.

[3] C. J. Damman, S. I. Miller, C. M. Surawicz, and T. L. Zisman, "The microbiome and inflammatory bowel disease: is there a therapeutic role for fecal microbiota transplantation," The American Journal of Gastroenterology, vol. 107, no. 10, pp. 14521459, 2012.

[4] P. B. Eckburg, E. M. Bik, C. N. Bernstein et al., "Microbiology: diversity of the human intestinal microbial flora," Science, vol. 308, no. 5728, pp. 1635-1638, 2005.

[5] R. E. Ley, C. A. Lozupone, M. Hamady, R. Knight, and J. I. Gordon, "Worlds within worlds: evolution of the vertebrate gut microbiota," Nature Reviews Microbiology, vol. 6, no. 10, pp. 776-788, 2008.
[6] S. Houston, G. W. Blakely, A. McDowell, L. Martin, and S. Patrick, "Binding and degradation of fibrinogen by Bacteroides fragilis and characterization of a $54 \mathrm{kDa}$ fibrinogen-binding protein," Microbiology, vol. 156, no. 8, pp. 2516-2526, 2010.

[7] J. L. Round, S. M. Lee, J. Li et al., "The toll-like receptor 2 pathway establishes colonization by a commensal of the human microbiota," Science, vol. 332, no. 6032, pp. 974-977, 2011.

[8] D. Moher, A. Liberati, J. Tetzlaff, and D. G. Altman, "Preferred reporting items for systematic reviews and meta-analyses: The PRISMA statement," International Journal of Surgery, vol. 8, no. 5, pp. 336-341, 2010.

[9] D. F. Stroup, J. A. Berlin, S. C. Morton et al., "Meta-analysis of observational studies in epidemiology: a proposal for reporting," Journal of the American Medical Association, vol. 283, no. 15, pp. 2008-2012, 2000.

[10] F. Brito, C. Zaltman, A. T. P. Carvalho et al., "Subgingival microflora in inflammatory bowel disease patients with untreated periodontitis," European Journal of Gastroenterology and Hepatology, vol. 25, no. 2, pp. 239-245, 2013.

[11] M. P. Conte, S. Schippa, I. Zamboni et al., "Gut-associated bacterial microbiota in paediatric patients with inflammatory bowel disease," Gut, vol. 55, no. 12, pp. 1760-1767, 2006.

[12] M. Mylonaki, N. B. Rayment, D. S. Rampton, B. N. Hudspith, and J. Brostoff, "Molecular characterization of rectal mucosaassociated bacterial flora in inflammatory bowel disease," Inflammatory Bowel Diseases, vol. 11, no. 5, pp. 481-487, 2005.

[13] F. C. Nwosu, L.-T. Thorkildsen, E. Avershina et al., "Agedependent fecal bacterial correlation to inflammatory bowel disease for newly diagnosed untreated children," Gastroenterology Research and Practice, vol. 2013, Article ID 302398, 7 pages, 2013.

[14] K.-L. Kolho, K. Korpela, T. Jaakkola et al., "Fecal microbiota in pediatric inflammatory bowel disease and its relation to inflammation," American Journal of Gastroenterology, vol. 110, no. 6, pp. 921-930, 2015.

[15] A. Andoh, H. Kuzuoka, T. Tsujikawa et al., "Multicenter analysis of fecal microbiota profiles in Japanese patients with Crohn's disease," Journal of Gastroenterology, vol. 47, no. 12, pp. 1298 1307, 2012.

[16] S. Ashorn, T. Honkanen, K.-L. Kolho et al., "Fecal calprotectin levels and serological responses to microbial antigens among children and adolescents with inflammatory bowel disease," Inflammatory Bowel Diseases, vol. 15, no. 2, pp. 199-205, 2009.

[17] C. Basset, J. Holton, A. Bazeos, D. Vaira, and S. Bloom, "Are Helicobacter species and enterotoxigenic Bacteroides fragilis involved in inflammatory bowel disease?" Digestive Diseases and Sciences, vol. 49, no. 9, pp. 1425-1432, 2004.

[18] B. Kleessen, A. J. Kroesen, H. J. Buhr, and M. Blaut, "Mucosal and invading bacteria in patients with inflammatory bowel disease compared with controls," Scandinavian Journal of Gastroenterology, vol. 37, no. 9, pp. 1034-1041, 2002.

[19] H. Sokol, P. Seksik, L. Rigottier-Gois et al., "Specificities of the fecal microbiota in inflammatory bowel disease," Inflammatory Bowel Diseases, vol. 12, no. 2, pp. 106-111, 2006.

[20] A. Swidsinski, A. Ladhoff, A. Pernthaler et al., "Mucosal flora in inflammatory bowel disease," Gastroenterology, vol. 122, no. 1, pp. 44-54, 2002.

[21] A. Swidsinski, J. Weber, V. Loening-Baucke, L. P. Hale, and H. Lochs, "Spatial organization and composition of the mucosal flora in patients with inflammatory bowel disease," Journal of Clinical Microbiology, vol. 43, no. 7, pp. 3380-3389, 2005. 
[22] H. Takaishi, T. Matsuki, A. Nakazawa et al., "Imbalance in intestinal microflora constitution could be involved in the pathogenesis of inflammatory bowel disease," International Journal of Medical Microbiology, vol. 298, no. 5-6, pp. 463-472, 2008.

[23] A. Andoh, H. Imaeda, T. Aomatsu et al., "Comparison of the fecal microbiota profiles between ulcerative colitis and Crohn's disease using terminal restriction fragment length polymorphism analysis," Journal of Gastroenterology, vol. 46, no. 4, pp. 479-486, 2011.

[24] R. K. Linskens, X. W. Huijsdens, P. H. M. Savelkoul, C. M. J. E. Vandenbrouckc-Grauls, and S. G. M. Meuwissen, "The bacterial flora in inflammatory bowel disease: current insights in pathogenesis and the influence of antibiotics and probiotics," Scandinavian Journal of Gastroenterology. Supplement, vol. 36, no. 234, pp. 29-40, 2001.

[25] P. Desreumaux and J.-F. Colombel, "Intestinal flora and Crohn's disease," Annales Pharmaceutiques Francaises, vol. 61, no. 4, pp. 276-281, 2003.

[26] A. Schwiertz, M. Jacobi, J.-S. Frick, M. Richter, K. Rusch, and H. Köhler, "Microbiota in pediatric inflammatory bowel disease," The Journal of Pediatrics, vol. 157, no. 2, pp. 240-244.e1, 2010.

[27] S. Matsumoto, N. Watanabe, A. Imaoka, and Y. Okabe, "Preventive effects of Bifidobacterium- and Lactobacillus-fermented milk on the development of inflammatory bowel disease in senescence-accelerated mouse P1/Yit strain mice," Digestion, vol. 64, no. 2, pp. 92-99, 2001.

[28] A. Swidsinski, V. Loening-Baucke, and A. Herber, "Mucosal flora in Crohn's disease and ulcerative colitis-an overview," Journal of Physiology and Pharmacology, vol. 60, supplement 6, pp. 61-71, 2009. 


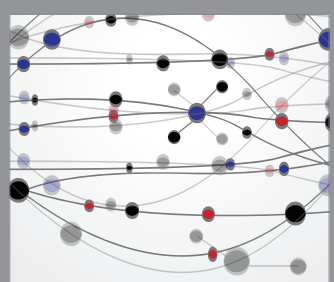

The Scientific World Journal
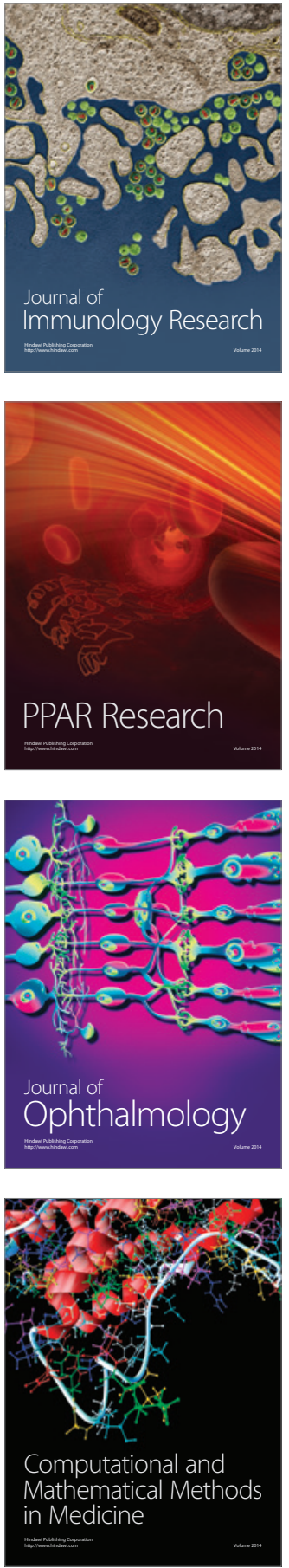

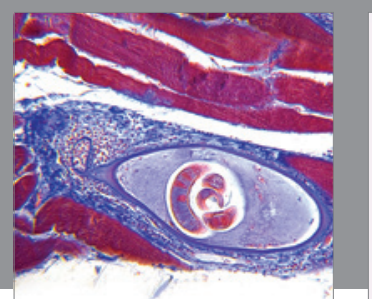

Gastroenterology Research and Practice

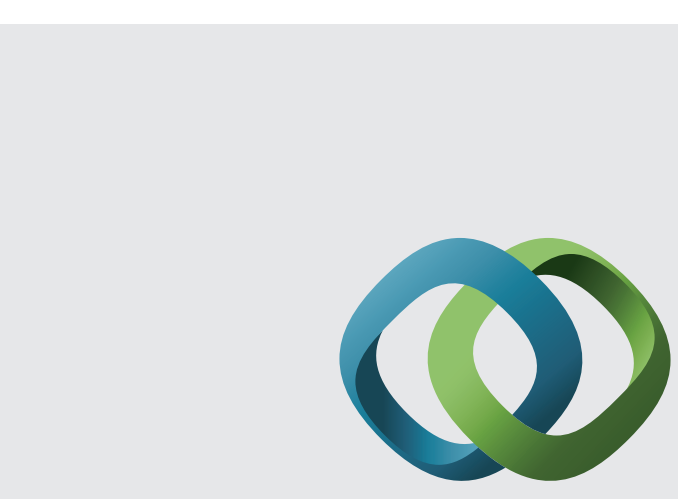

\section{Hindawi}

Submit your manuscripts at

http://www.hindawi.com
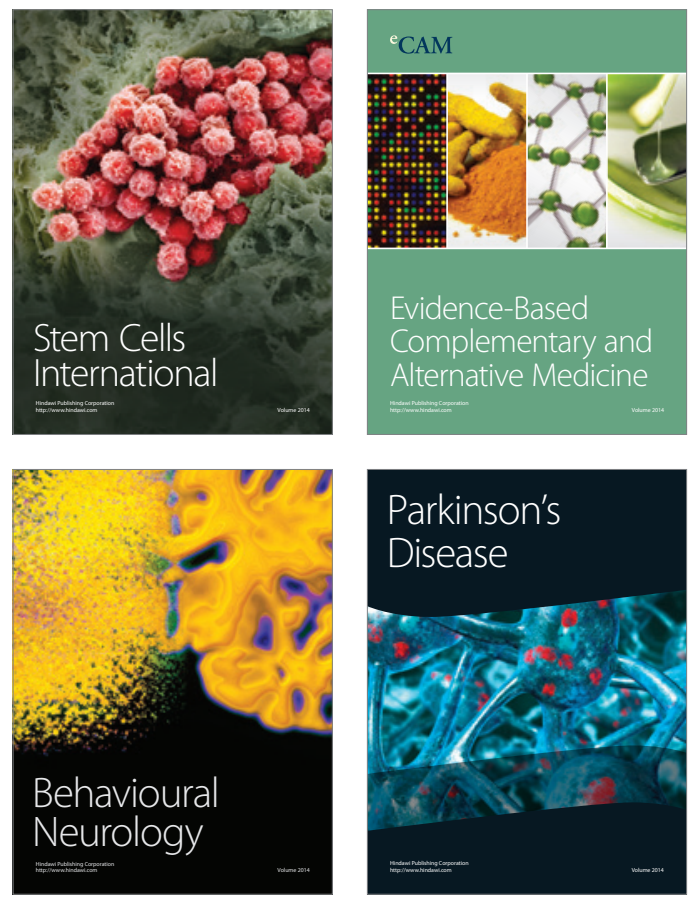
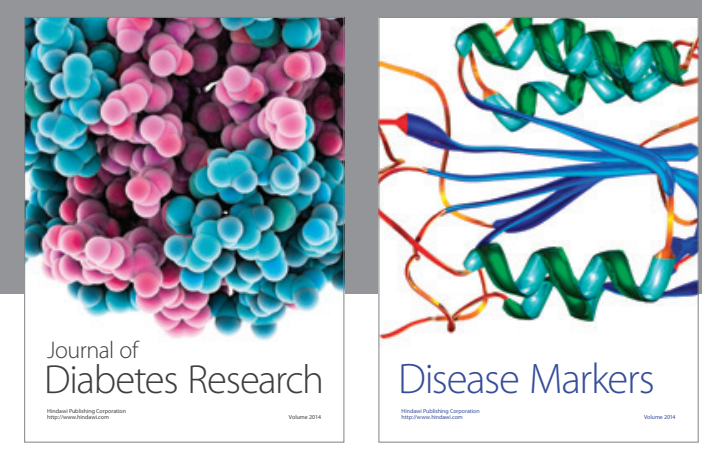

Disease Markers
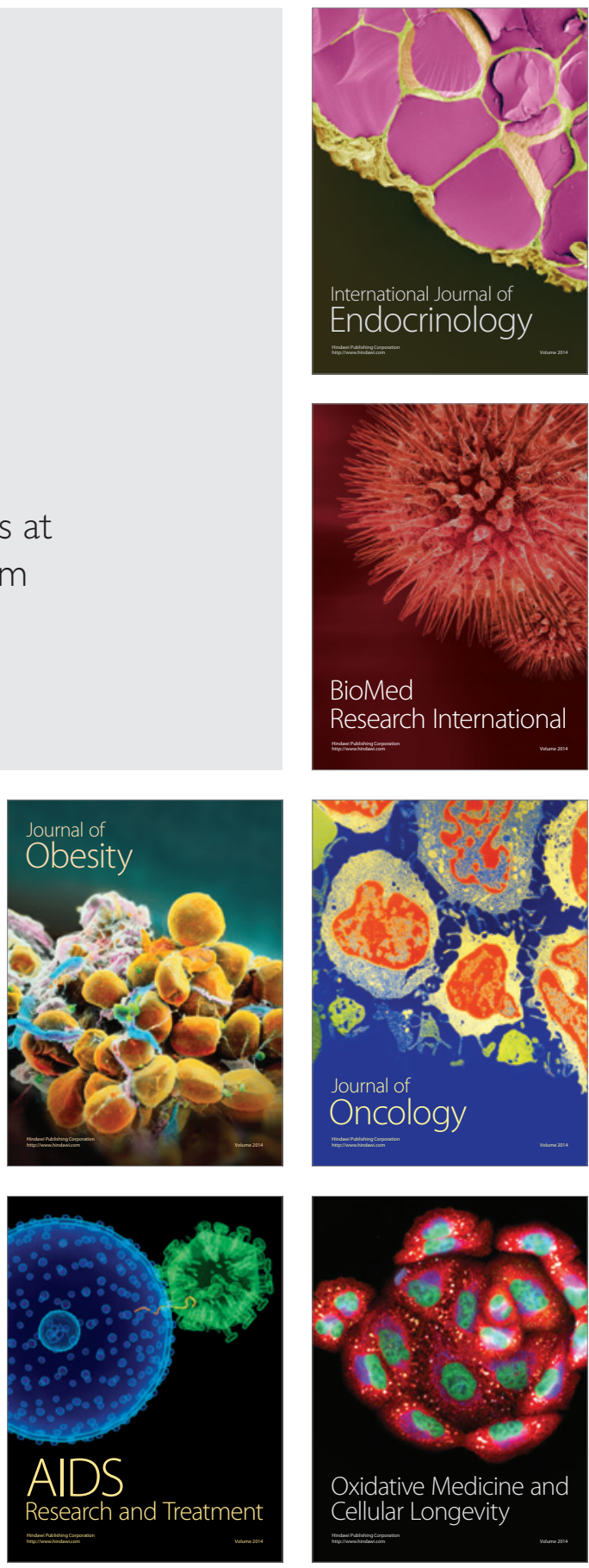